\title{
CREATION OF PNEUMOPERITONEUM BY A NEW TECHNIQUE PRIOR TO LAPAROSCOPIC PROCEDURE
}

\author{
Atta Hussain Soomro
}

\begin{abstract}
OBJECTIVE: To evaluate the safe and easy technique for establishing the pneumoperitoneum, which best suits to our needs and compare it with other available techniques.

DESIGN: A descriptive study

SETTING: This study was conducted in Larkana, Sindh from February 2002 to July 2003.

PATIENTS AND METHODS: A total of 376 patients underwent different laparoscopic procedures.

The pneumoperitoneum was created with veress needle in 80 patients, open technique was used in 20 patients while in the remaining 276 patients a new technique was used.

RESULTS: The time used for creation of pneumoperitoneum with veress needle was 5 minutes, by open method 8 minutes and by new technique only one minute and 30 seconds. The rate of complications with veress needle was $1.25 \%$ (one patient), with open technique $10 \%$ (two patients) and with new method only $0.724 \%$ (two patients). All the complications were minor injuries to omentum or mesentry or serosa of the small bowel which did not require any treatment or repair.

CONCLUSION: The advantages seen by this new technique are; easy to perform, less time consuming, safe with minimum possible complications and do not requiring any additional cost. It is recommended as a best available method for insufflation in laparoscopy, particularly suitable to our needs.
\end{abstract}

KEY WORDS: Pneumoperitoneum. Laparoscopy.

\section{INTRODUCTION}

Minimal invasive surgery has a considerable impact in common surgical techniques, particularly in laparoscopic cholecystectomy and appendectomy. ${ }^{1}$ At present, there are many methods being used for insufflation prior to laparoscopy. One method is blind veress needle/trocar insertion; other is open Hasson trocar or modified blunt trocar insertion. Third method is the use of optiview visualizing trocar. There is also a new method which focuses on direct trocar insertion.

This study evaluates the safe and quicker method for creation of pneumoperitoneum with the aim that which of these methods is suitable to needs in our setup.

\section{PATIENTS AND METHODS}

A total of 376 consecutive patients underwent different laparoscopic procedures. Pre-operatively, these patients had routine investigations for diagnosis and fitness for general anesthesia. Carbon dioxide gas was used for insufflation. The procedures used for creation of pneumoperitoneum were; the veress needle/trocar insertion in 80 patients, open method in 20 patients and new technique in 276 patients. These three were performed as mentioned in the literature but here we would like to mention the last one i.e. the new technique. For this a $10 \mathrm{~mm}$ vertical/transverse incision is made below the umbilicus (Figure I) except to those patients who have previous lower abdomen surgery. Anterior abdominal wall is pulled up from both sides of umbilicus so that the parietal peritoneum is separated from the viscera (Figure II). Now a 10 $\mathrm{mm}$ trocar is introduced through the incision without application of much force till the tip of trocar reaches the linea alba (Figure III). When a resistance is felt a gentle soft pressure is applied here so that the tip of trocar enters the linea alba. The trocar is then removed (Figure IV) and with soft pressure on cannula which entered into the peritoneal cavity (Figure V). The cannula is now connected to the insufflating gas source and pneumoperitoneum is obtained. 
Figure I: Application of transverse incision just below the umbilicus

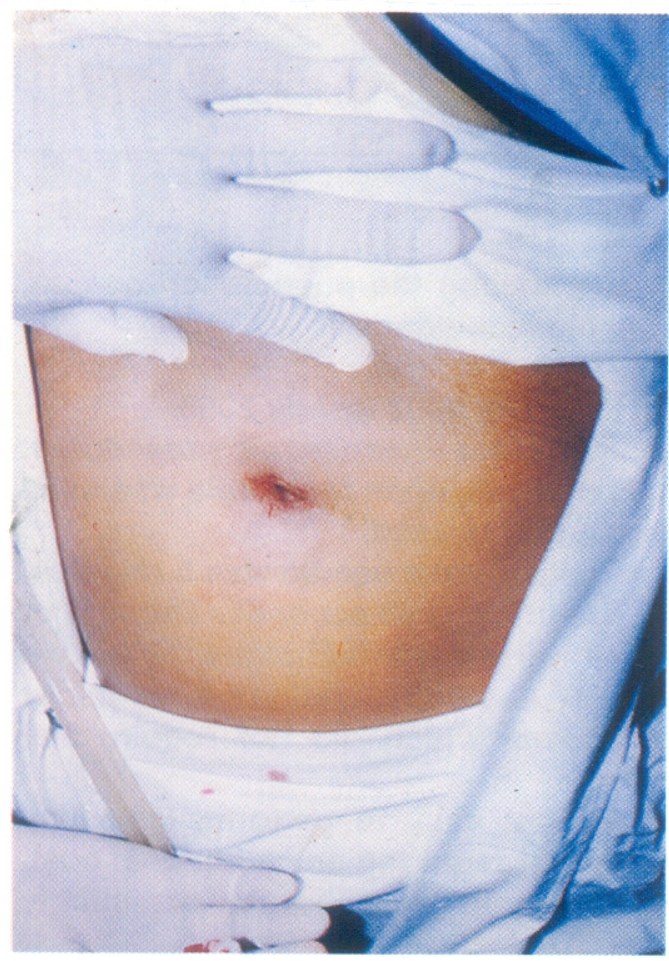

Figure II: Elevation of anterior abdominal wall so as to separate the partial peritoneum from the viscera and $10 \mathrm{~mm}$ trocar is ready for insertion

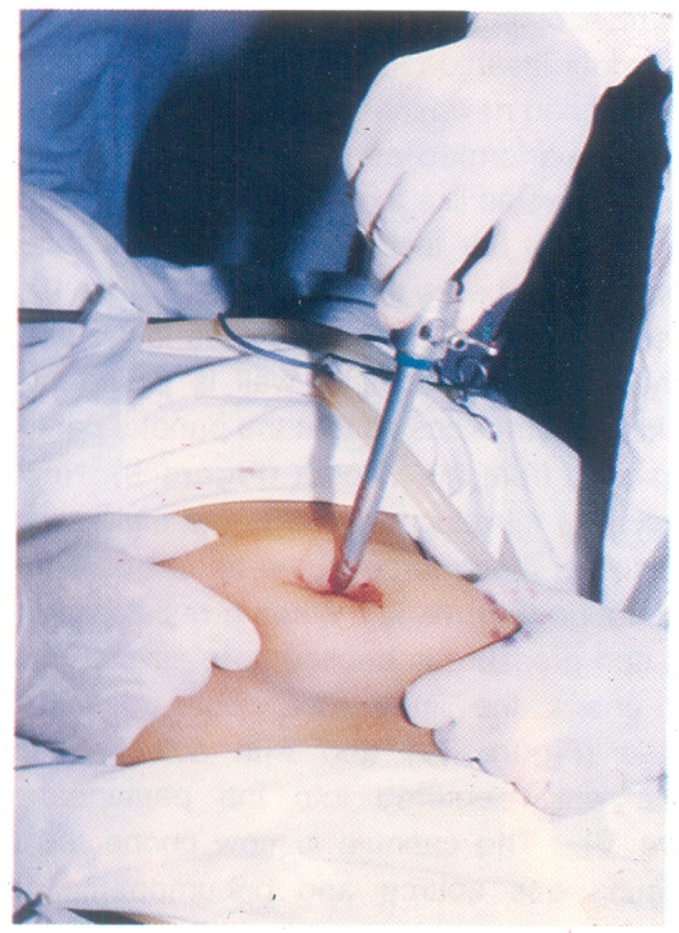

Figure III: Introduction of trocar till the rectus sheath

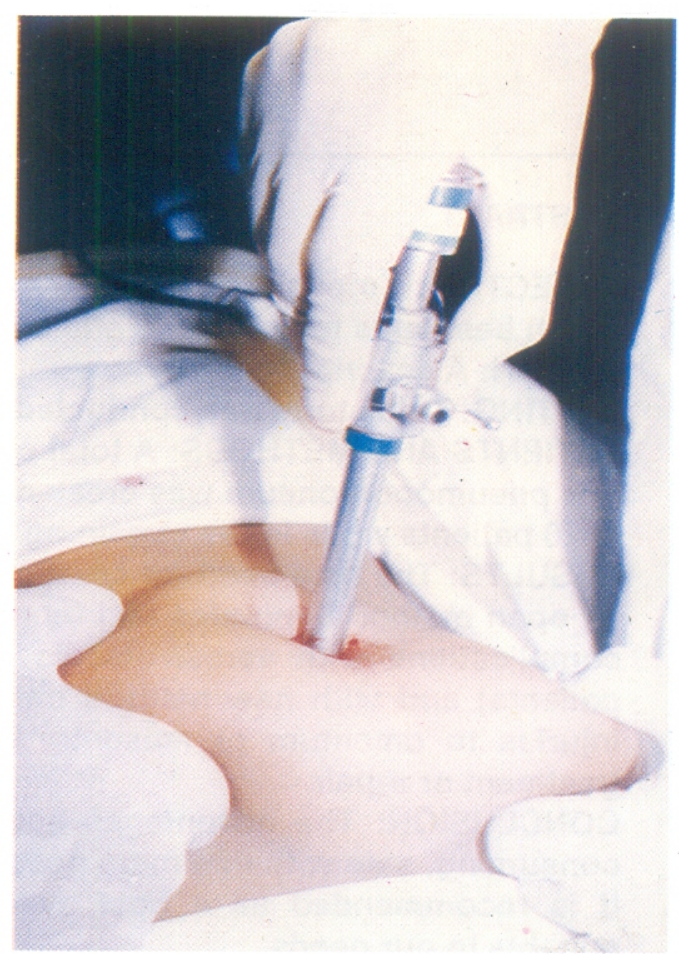

Figure IV: The trocar of cannula is pulled up so as to prevent injury of the visceras

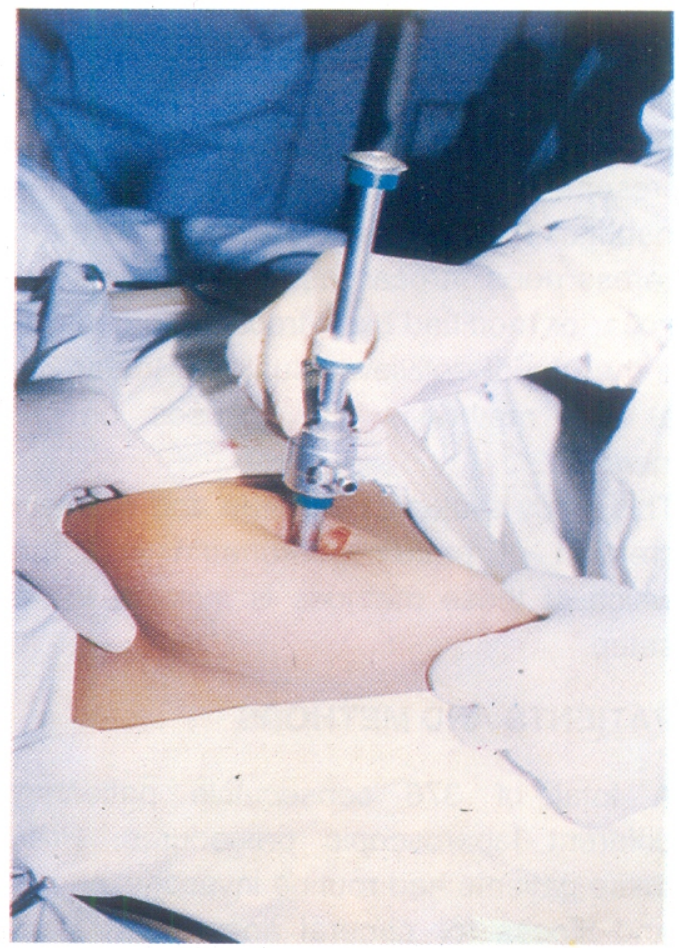


Figure V: Remove the trocar, now push the cannula entirely inside the peritoneal cavity (till now the anterior abdominal wall is pulled up). Now connect with insufflator

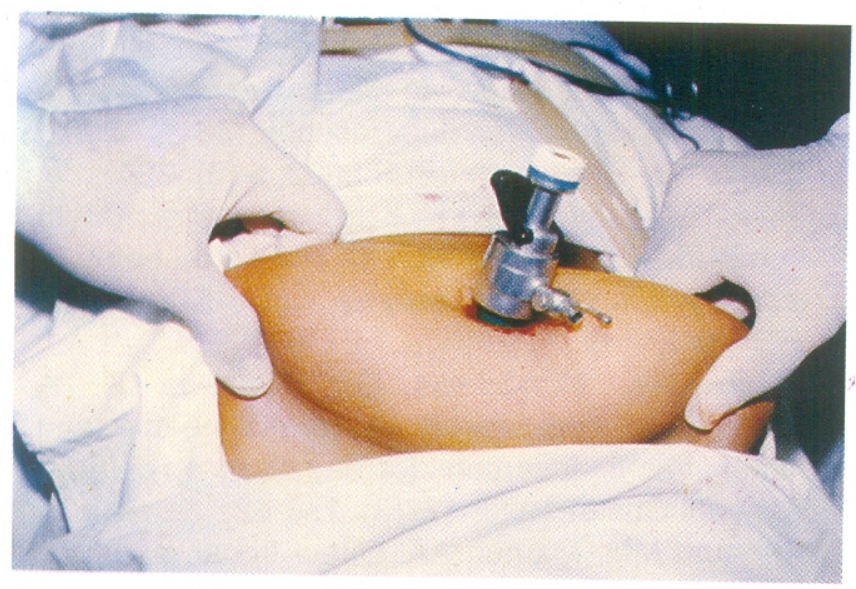

\section{RESULTS}

Among 376 patients, 80(21.32\%) underwent veress needle method for creating pneumoperitoneum, 20 (5.32\%) open method and the remaining 276 (73.4\%) underwent this new technique by means of direct trocar insertion for insufflation. Male and female ratio was 1:2.2. The age ranged from 10-80 years ( average 45 years).

The time taken for creation of pneumoperitoneum in veress needle was 5 minutes, in open method 8 minutes and by this new method it was .1 minute and 30 seconds. The rate of complications with veress needle was $1.2 \%$ ( 1 patient), with open technique $10 \%$ (2 patients) and with this new technique, it was only $0.72 \%$ ( 2 patients). In veress needle method the complications were omental injury in one patient and serosal tear of small bowel in other patient while this new technique had also 2 complications; serosal injury of small bowel in one patient and injury of mesentry in another. These injuries were of minor nature and did not require any repair.

\section{DISCUSSION}

Different techniques of establishing pneumoperitoneum for laparoscopy are available. Some of these are in common use and others are being evaluated. The commonly used technique is blind insertion of veress needle/trocar but this method has been substantially criticized in the literature for being a blind procedure and subjected to complications. Potential danger of veress needle blind insertion is the occurrence of visceral or vascular injury. ${ }^{2}$ It also has been reported as dangerous for intra abdominal vessels. ${ }^{3}$ The other method is open technique using Hasson's trocar or modified Hasson method. Different comparative studies have recommended the open method. ${ }^{4}$ One comparative study of open method versus veress needle has indicated open access towards a reduced risk of access site herniation and reduced risk of minor complications ${ }^{5}$. In another comparative study between open and closed technique, open technique has been shown to be safer with low risk of early and late complications. ${ }^{6}$ Meanwhile other comparative studies have recommended the open procedure with modified Hasson trocar. ${ }^{7-9}$

Though this new technique for pneumoperitoneum creation is performed by direct trocar insertion with reusable trocar of $10 \mathrm{~mm}$ which is safe alternative to veress needle insertion with low rate of complications. This approach has further advantages such as less cost or instrumentation and rapid creation pneumoperitoneum. ${ }^{10}$ In entering the abdomen directly with a trocar, critical surgical points are emphasized such as; adequate relaxation, sharp trocars, adequate skin incision, elevation of abdominal wall and insertion of tocar into the true pelvic cavity.

Copeland et al suggest that this technique offers more clinical security because it does not place reliance on secondary tests but emphasize concentration entirely upon surgical skill and anatomic knowledge during the entry. ${ }^{8}$ In a comparative study of this method versus veress needle technique, Byron et al prefer the direct insertion technique for trocar insertion versus veress needle technique due to fewer minor complications and less operating time. They report mean laparoscopic insertion time of 2.2 minutes and 5.9 minutes for the direct insertion and veress needle technique respectively. ${ }^{11}$ Other studies have also recommended the direct trocar techniqure as safe approach to abdominal entry for laparoscopic surgery. ${ }^{9,12}$ Vakili has claimed that this manual provides much better visceral and tactile control and is done more expeditiously particularly in obese patients. ${ }^{13}$ We have also shown in our study that direct trocar insertion technique is better than the closed or open methods because of its advantages such as; it is easy to perform, less time consuming, safe with minimum possible complications and does not requiring any additional cost.

\section{CONCLUSION}

Our experience shows that this new technique is a superior method for creation of pneumoperitoneum, particularly suitable to our needs as it is cost effective, with rapid creation of pneumoperitone.um, easy to perform and safe with few minor complications. 


\section{REFERENEES}

1. Alvarez C, Votik AJ. The road of ambulatory laparoscopic management of perforated appendicitis. Am J Surg 2002; 179(1): 63-6.

2. Bonjer HJ, Hazebroek EJ, Kazemier G et al. Open versus closed establishment of pneumoperitoneum in laparoscopic surgery. $\mathrm{Br} \mathrm{J}$ Surg 1997; 84(5): 599-602.

3. Qureshi FA. Anesthesia related complications of laparoscopic cholecystectomy. JCPSP 2003; 13 (7): 369-71.

4. Soomro AH, Memon AA, Dholia KR et al. An audit of 45 laparoscopic appendectomies. JtUMHS 2003; 2 (1): 18-20.

5. Merlin TL, Hiller JE, Maddern GJ et al. Systematic review of the safety and effectiveness of method used to establish pneumoperitoneum in laparoscopic surgery. Br J Surg 2003; 90(6): 668-79.

6. McKernan JB, Champio JK. Access technique: veress needle-initial blind trocar insertion versus open laparoscopy with Hasson trocar. Endosc Surg Altied Technol 1995; 3(1): 35-8.

7. Marcovich R, Del Terzo MA, Wolf JS Jr. Comparison of transperitoneal laparoscopic access techniques: optiview visualization and veress needle. Endourol 2000; 14(2): 175-9.

8. Copeland C, Wing R, Hulka JF. Direct trocar insertion at laparoscopy: an evaluation. Obstet Gynecol 1983; 62(5): 655-9.

9. Jacobson MT, Osias J, Bizhang $R$ et al. The direct trocar technique: an alternative approach to abdominal entry for laparoscopy JSLS 2002; (2):167-74. Erratum in JSLS 2002; 6 (3): 224.

10. Yerdel MA, Karayaclin K, Koyuncu A et al. Direct trocar insertion verses veress needle insertion in laparoscopic cholecystectomy. Am J Surg 1999; 177 (3): 247-9.

11. Byron JW, Markenson G, Miyazawa K. A randomized comparison of veress needle and direct trocar insertion for laparocscopy. Surg Gynecol Obstet 1993; 177(3): 259-62.

12. Rahman MM, Mamun AA. Direct trocar insertion: alternative abdominal entry technique for laparoscopic surgery. Mymensingh Med J 2003; $12(1): 45-7$.

13. Vakili C, Knight R. A technique for needle insufflation with obese patients. Surg Laparosc Endosc 1993; 3 (3): 489-91.

AUTHOR AFFILIATION AND CORRESPONDENCE ADDRESS:

\section{Dr. Atta Hussain Soomro}

Assistant Professor, Department of Surgery, Chandka Medical College, Larkana, Sindh, Pakistan.

E-mail: atta_cme@yahoo.com 